\title{
UNIVERSITYOF
}

FORWARD

THINKING

WESTMINSTER用

WestminsterResearch

http://www.westminster.ac.uk/westminsterresearch

A Survey of the Current Status of Research on Quantum Games

Huang, D. and Li, Shuliang

This is a copy of the author's accepted version of a paper subsequently published in the proceedings of the 2018 4th International Conference on Information Management (ICIM2018) IEEE, The University of Oxford, Oxford, United Kingdom, 25 to 27 May 2018.

The final published version is available online at:

https://doi.org/10.1109/INFOMAN.2018.8392807

C 2018 IEEE . Personal use of this material is permitted. Permission from IEEE must be obtained for all other uses, in any current or future media, including reprinting/republishing this material for advertising or promotional purposes, creating new collective works, for resale or redistribution to servers or lists, or reuse of any copyrighted component of this work in other works.

The WestminsterResearch online digital archive at the University of Westminster aims to make the research output of the University available to a wider audience. Copyright and Moral Rights remain with the authors and/or copyright owners.

Whilst further distribution of specific materials from within this archive is forbidden, you may freely distribute the URL of WestminsterResearch: ((http://westminsterresearch.wmin.ac.uk/)).

In case of abuse or copyright appearing without permission e-mail repository@westminster.ac.uk 


\section{A Survey of the Current Status of Research on Quantum Games}

\author{
Dingxuan Huang 1,2 \\ ${ }^{1}$ School of Management \\ Guilin University of Technology \\ Guilin 541004, China \\ huangdingxuan@126.com \\ ${ }^{2}$ Modern Enterprise Management Research Center, \\ Guilin University of Technology, Guilin 541004, \\ China.
}

\author{
Shuliang $\mathrm{Li}^{3,4}$ \\ ${ }^{3}$ Westminster Business School \\ University of Westminster \\ London NW1 5LS, United Kingdom \\ s.li@westminster.ac.uk \\ ${ }^{4}$ School of Economics \& Management \\ Southwest Jiaotong University \\ Chengdu, Sichuan 610031, China
}

\begin{abstract}
Quantum games have gained considerable interest from researchers. In this paper, on the basis of the Web of Science database, through the use of the social network analysis methods, the literature on quantum games is analyzed from three aspects: the keywords co-occurrence, coauthorship, and co-citation. In the process of analysis, the main quantum game models are reviewed with graphical illustrations. Our paper provides a survey and outline of the current status of research in this field, and identify directions for future work.
\end{abstract}

Keywords - quantum game; quantum strategy; game model; social network

\section{INTRODUCTION}

The game theory plays an important role in decision science and can be widely applied in nearly every aspect of our modern society. In recent few years, along with the thriving of quantum computation and quantum information, a newly emerging area, quantum game has begun to attract much attention due to the intimate connection between the theory of games and the theory of quantum communication [1]. Quantum games are initiated by the papers from Meyer and Eisert et al. Almost at the same time, Meyer's finding shows that a player can always beats his classical opponent by adopting quantum strategies on the coin tossing game; Eisert et al. Find that the contradiction exists in the classical scenario vanishes by introducing quantum entanglement into the famous Prisoners' Dilemma ${ }^{[2,3]}$. After that, comparing with their classical counterpart game models, the quantum game theory has exhibited great superiority and different characters ${ }^{[4-7]}$. It was used to investigate the social problem and market features ${ }^{[8,9]}$.

In order to understand relevant ideas, our paper seeks to introduce and provide a summary and outline of quantum games. Towards this aim, the content is organized as following: Sections 2 is the introduction to the source of research papers and research tools. In section 3, the social network characteristics of research samples are analyzed and presented. In Section 4, the main models on quantum game research are shown and illustrated. Section 5 provides the concluding remarks and envisages future research in this area.

\section{SAMPLE SECTION AND SOFTWARE}

Aiming at confirming to the principles of objectivity, the databases of Web of Science are chosen as the source of references. The reason lies in two. Firstly, the Web of Science is one of the largest Web-based research databases sin the world and has been widely used. Secondly, the downloaded references from this database include titles, keywords, authors, abstracts and references. This helps to analyze the social network of the references, especially the co-citations and cited authorship.

During the process of reference selection, the core collection of the Web of Science is selected as the source. The type of publications is only "article". And "quantum game" is used as the title. The end of time period is set as $30^{\text {th }}$ June 2017. In total, 254 references are chosen as research samples. In order to ensure the accuracy of data analysis, some pre-processing must be done. For example, "quantum game" and "quantum games" in keywords should be unified into quantum game. Bibexcel and VOSviwer are use as software tools to help realize the social network analysis.

\section{SOCIAL NETWORK OF QUANTUM GAME RESEARCH}

A social network is defined as a finite set or sets of actors and the relationship or relationships between them. The interrelations of actors may include friends, relatives, classmates, and colleagues, and each actor develop and build his or her own style of social networking ${ }^{[10]}$. As an advanced and robust technique, SNA is suitable for analyzing the social network behaviors in quantum game research and can provide a more relational, contextual, and holistic picture.

\section{A. The co-occurrence of author keywords and keywords} plus

There are usually only $3-5$ key words in one paper. But these key words are highly refined and summarized from the contents, reflecting a core theme. Therefore, the co- 
occurrence of author keywords related to research on quantum games can reflect the frontier topics in this area.

In order to find the co-occurrence characteristics of keywords from two different aspects, we choose the author keywords and the keywords plus for our analysis. Keywords plus is added to the traditional keyword retrieval or title, reflected in the author's paper title index. The minimum number of occurrences of author keywords is 3. Thus, 29 author keywords meet the threshold, among the 352 keywords in the 254 papers. For each of the 29 author keywords, the total strength of the co-occurrence linking with other keywords will be calculated. The keywords with the greatest total link strength will be selected. The overlapping visualization of author keywords is shown in Fig.1. It is very clear that the main author keywords related to research on quantum games include game theory, Nash equilibrium, entanglement, decoherence, quantum strategies. The major co-occurrence includes the quantum game and

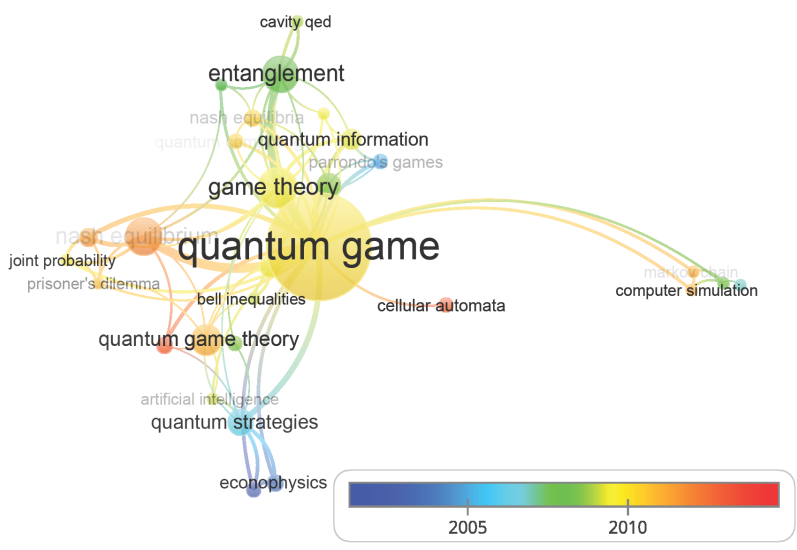

Figure 1. Overlapping visualization of author keywords

Nash equilibrium, entanglement and the quantum game, the quantum game and quantum probability, the quantum game and minority game. In figure 1 , we can see the change of keywords over time. In this picture the color of keywords varied over time.

In order to further verify the theme of research on quantum game theory, keywords plus is adopted to verify the research topic from another view. The visualization of keywords plus is illustrated in figure 2, which shows that decoherence, entanglement, cooperation and information are the top keywords plus. On the other hand, the variation of paper titles over time can basically be discern in figure 2 . In this picture, the color of keywords plus varied over time.

\section{B. The co-authorship of authors}

The co-authorship of authors reflects the objective relation between the authors, and reveals the organizational structure of researchers and research directions in an area.

During the analysis of the co-authorship of authors, the
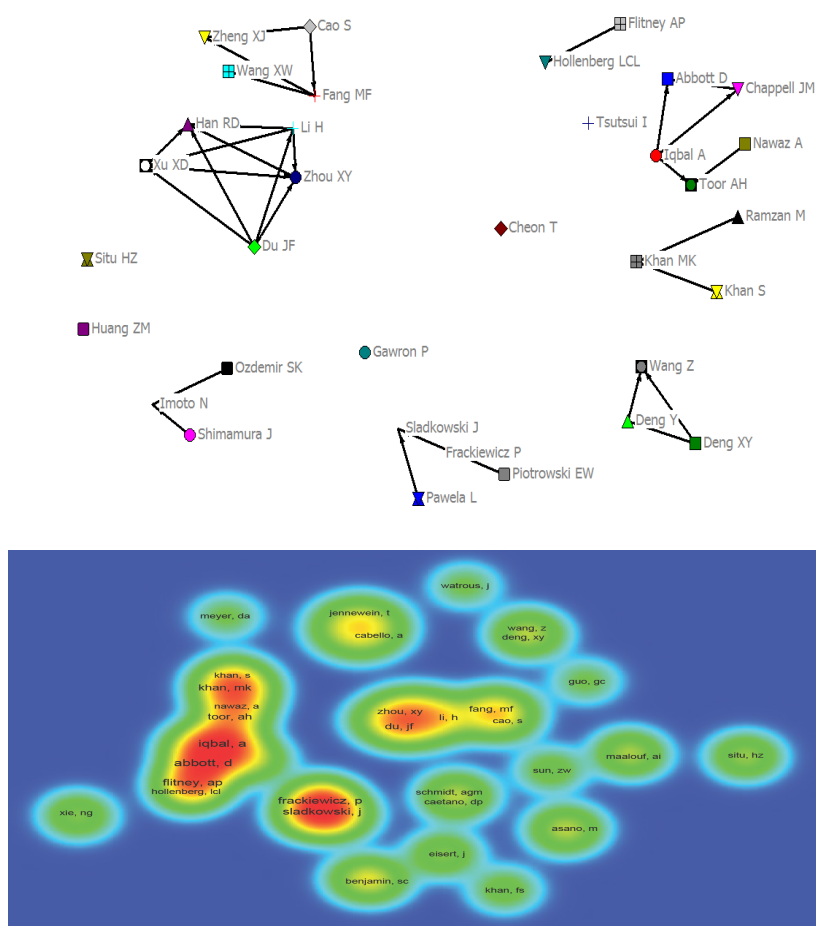

Figure 4. Density visualization of co-authorship

minimum number of documents of an author is 3. Among the 458 authors, 59 of them meet this requirement. Iqbal $\mathrm{A}$ is the author who appear 20 times. Abbott D is the second with 13 times. Frackiewicz $\mathrm{P}$ ranks third, with 12 times. And Sladkowski J comes next, with 11 times. The authors with top five ranking co-authorships are given as follows. Iqbal A and Abbott D have 11 times; Ramzan $\mathrm{M}$ and Khan $\mathrm{M}$ K have 8 times; Chappell JM and Abbott D have 7 times; Iqbal A and Toor AH have 6 times; Du JF and Li H, Khan S and Khan MK, and Chappell JM and Iqbal A have 5 times. The social networks on the co-authorship are shown in figure 3 and figure 4.

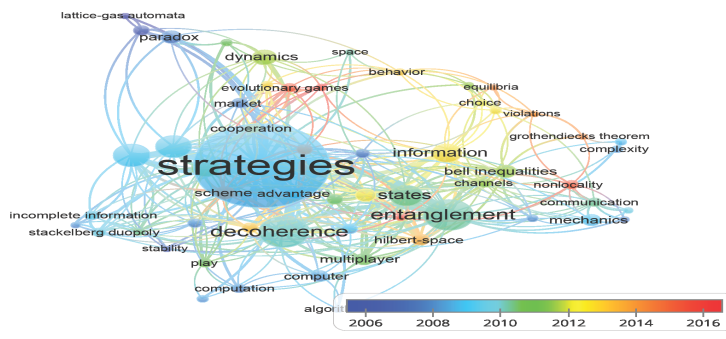

Figure 2. Density visualization of keywords plus 
TABLE 1. TOP 10 CO-CITATION OF CITED REFERENCES AND CITED SOURCES

\begin{tabular}{|c|c|c|c|c|}
\hline Rank & Cited reference & Citations & Cited source & Citations \\
\hline 1 & Eisert j, 1999, PHYS REV LETT & 190 & PHYS REV LETT & 916 \\
\hline 2 & Meyer da, 1999, PHYS REV LETT & 159 & PHYS REV A & 682 \\
\hline 3 & Marinatto 1, 2000, PHYS LETT A & 103 & PHYS LETT A & 674 \\
\hline 4 & Benjamin sc, 2001, PHYS REV A & 88 & J PHYS A-MATH GEN & 218 \\
\hline 5 & Eisert j, 2000, J MOD OPTIC & 74 & PHYSICA A & 202 \\
\hline 6 & Du jiangfeng, 2002, PHYS REV LETT & 74 & NATURE & 173 \\
\hline 7 & Johnson nf, 2001, PHYS REV A & 47 & J PHYS A-MATH THEOR & 129 \\
\hline 8 & Nielsen m. A., 2000, QUANTUM COMPUTATION & 45 & QUNTUM INF PROCESS & 117 \\
\hline 9 & Flitney ap, 2005, J PHYS A-MATH GEN & 45 & J MOD OPTIC & 100 \\
\hline 10 & Benjamin sc, 2001, PHYS REV LETT & & 90 \\
\hline
\end{tabular}

At least three research teams can be found in figure 3 . The first research team includes Iqbal A, Toor AH, Chappell JM, Abbott D and Nawaz A. The members of the second team are $\mathrm{Du}$ JF, Han RD, Xu XD, Li $\mathrm{H}$ and Zhou XY. The third research team includes Wang XW, Fang MF, Cao $\mathrm{S}$, and Zheng XJ. Several other research teams seem to be in the formation process. Relevant examples are Shimamura J and Imoto, Khan S and Khan MK and Ramzan M, etc. Density visualization of co-authorship is presented in figure 4, which shows the intensity of collaboration between different authors by using different colors. The more pronounced the color is, the more times the author appears.

\section{The co-citation of cited reference and cited sources}

Analysis on co-occurrence of cited reference can achieve the following two objectives. Firstly, to evaluate periodicals and papers and investigate the law of reference flows.

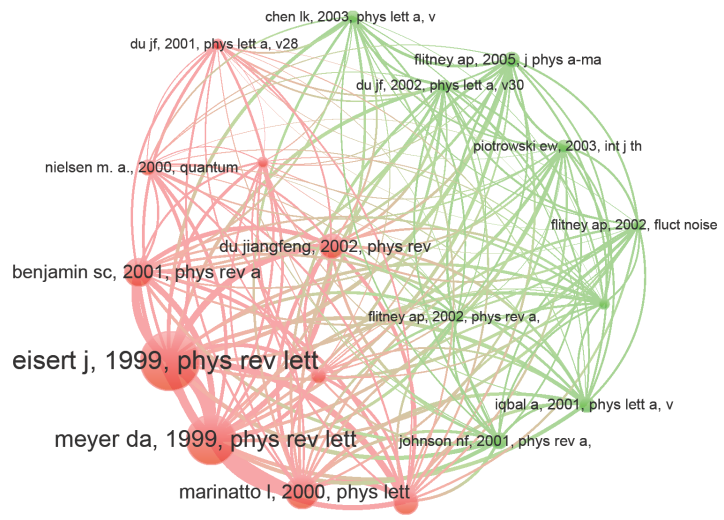

Figure 5. Co-citation of cited reference

Secondly, to gain insights into the development and connection of disciplines, and analyze the trend and directions of the research area.

The minimum number of citations of a cited reference is 20. Among the 3310 cited references, only 38 meet this requirement. Also, there are 688 co-citations. Furthermore, we selected 19 articles that were cited more than 30 times. And 171 co-citations occurred from these articles. The findings are shown in figure 5. It is clear that pioneering papers by Meyer and Eisert et al are widely cited by peer researchers. Their work have been cited 190 and 156 times, respectively. The co-citation of the cited source is used to study the main research magazines that published the famous papers on quantum games. The result is shown in figure 6. It suggest that the magazine PHYS REV LETT, PHYS REV A

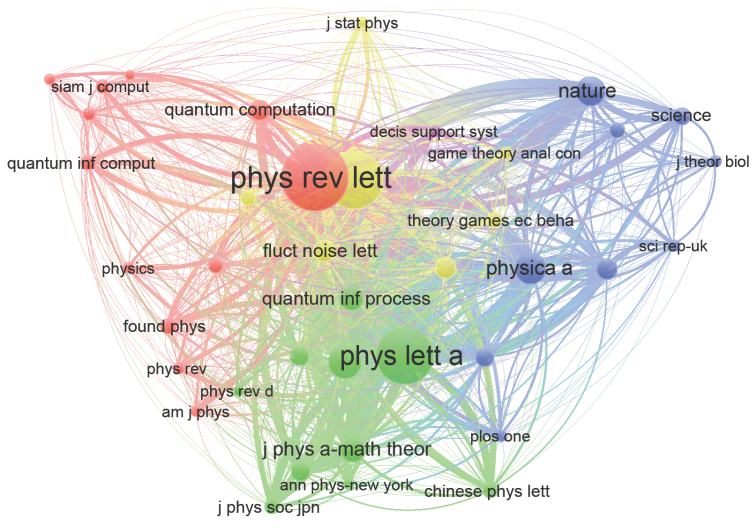

Figure 6. Co-citation of cited sources

and PHYS LETT A are the top 3 journals. Detailed information about the top 10 co-citations of cited references and cited sources is given in table 1 .

\section{MAIN MODELS OF QUANTUM GAMES AND APPLICATIONS}

Various types of quantum games have been studied, including those classic cases typically encountered in game theory courses. Most of the initial results showed that quantum games offered ways around classic dilemmas [11] Several research directions on quantum game research emerged. Many researchers focused on the representation of quantum games and counterpart classical game models. Others, such as Nash equilibria, looked into theoretical properties of quantum games or evolutionary games. Finally, a number of researchers opened new application areas of quantum game theory in market research and society managements. In this paper, one of the tasks is to summarize 
the characteristics of the most important quantum game models.

\section{A. The main classical game models and its quantum counterpart}

In the research on quantum game, penny flip or coin flip is a game that two players take turns in flipping an electron up or down twice and then the electron's final state is measured. Meyer brought Penny flip into quantum games. he shows, in his paper, that a player who implements a quantum strategy can enhance his expected payoff and confirms that, generally, a quantum strategy is always at least as good as a classical one ${ }^{[3]}$. Romanelli and Hernandez explored the global chirality distribution of the quantum walk on the line when decoherence is enclosed, which is similar to the spin-flip game. These games are characterized by a continuous space of strategies, and the selection of an initial condition determines the particular strategy chosen by the player ${ }^{[12]}$. Iqbal and Abbott use the setting of a quantum correlation experiment for considering the instances of matching pennies ${ }^{[13]}$.

Prisoners' game is a game that two players select and decide either to admit a crime or to deny a crime (corporate with each other), their payoff from cooperation is better than the payoff when they do not cooperate. Eisert et al. investigate the quantization of nonzero sum games and show that this game stops presenting a dilemma if quantum strategies are accepted [2]. By using the Eisert quantum games, Flitney and Hollenberg show that different Nash equilibrium with different classical-quantum transitions can appear ${ }^{[14]}$. Iqbal et al. find a quantum version of two-stage repeated classical game of prisoners' dilemma, where the players choose to cooperate in the first stage while knowing that both will defect in the second ${ }^{[15]}$. Iqbal et al. extend joint probabilities in the EPR-Bohm setting to demonstrate general three-player non-cooperative symmetric games. Their findings about the three-player generalized Prisoner's Dilemma (PD) shows that the players can run away from the classical outcome of the game ${ }^{[16]}$.

Battle of the Sexes is another important model. It is found that quantization cannot resolve the dilemma completely for the Battle of the Sexes, while it, in general, can do for the Prisoners' Dilemma and Stag Hunt if appropriate correlations for the strategies of the players are given. Briefly, quantum entanglement can deal with classical dilemmas for some games, and the games for which this is possible can be judged from the classical payoff matrices [17]. Marinatto and Weber extend the concept of a classical two-person static game to the quantum domain, by giving an Hilbert structure to the space of classical strategies and investigating the Battle of the Sexes game. They show that the introduction of entangled strategies leads to a unique solution of this game ${ }^{[18]}$.

Quantum Cournot's duopoly are introduced by $\mathrm{Li} \mathrm{H}$ et al. Li Y et al., Zhou $J$ et al. Some novel features in the quantum Cournot's duopoly are observed, which are completely due to quantum entanglement. For instance, the total profit at the Nash equilibrium always gains maximum once the von Neumann entropy tends to infinity. The quantum entanglement enables an arbitrary number of players to cooperate to the biggest extent $[1,19,20]$.

Minority game is a game that widely used in brand management and stock market research. It shows that players win if their strategy is in the minority group with an odd number of players have two strategies. Chen et al. investigate the $\mathrm{N}$-player quantum minority game and find that all pure quantum strategies just decrease to classical ones when $\mathrm{N}$ is an odd number. When $\mathrm{N}$ is even, basically non-classical equilibrium become available and the players can get better expected payoffs at the same time ${ }^{[21]}$. Flitney and Greentree consider the advantage obtained in a quantum minority game by a coalition sharing an initially entangled state versus that obtained by a coalition that uses classical communication to reach an optimal group strategy. Their result shows that quantum coalitions outperform classical ones when carried out by up to four players ${ }^{[22]}$. Flitney et al. show that, for a continuous set of entangled four-partite states, the task of maximizing the payoff in the symmetric strategy four-player quantum minority game is equivalent to maximizing the violation of a four-particle Bell inequality ${ }^{[23]}$. Furthermore, Hill and Flitney construct a competitive game between four players based on the minority game where the maximal Nash equilibrium payoff when played with the appropriate quantum resource is higher than that obtainable by classical means, assuming a local hidden variable model ${ }^{[24]}$. Based on a multiple-users multiple-choice quantum minority game, Zabaleta et al. propose a model for managing spectrum fairly and effectively, by taking advantage of quantum entanglement and quantum interference. It is possible to lessen the chance of collision problems associated with classic algorithms ${ }^{[25]}$.

Parrondo's game or Parrondo's paradox arises when two losing games are integrated to generate a winning one [26] Flitney et al. have proposed a protocol for a quantum version of a history-dependent Parrondo's game. If the initial state is a superposition, payoff difference from the classical game can be acquired as a result of interference [27]. Chen et al. develop a quantum version of Parrondo's game based on a quantum ratchet effect for a delta-kicked model. And a quantum anti-Parrondo's game is also investigated in their paper ${ }^{[28]}$. Moreover, Parrondo's game combined with Markov chain or quantum walk are investigated. These results show that the explanation of the game related to a stationary probability distribution is that the probability of the initial capital has achieved parity [6, 29-32].

Evolutionary stable strategy (ESS) is the kernel concept in evolutionary game theory, which is delineated as a strategy that cannot be attacked by mutant strategies once fixed in the population. Evolutionary quantum games were introduced by Iqbal et al, Kay R et al. ${ }^{[33,34]}$. It shows that, in 
certain types of games, entanglement can be applied to enable appear or disappear ESSs, while keeping associated Nash equilibrium. On the other hand, a quantum evolutionary game dominates a classic game if time steps are big. Nevertheless, the quantum evolutionary game is not better with small time intervals. Furthermore, in the evolutionary public goods game, entanglement can also contribute to the persistence of cooperation under various population structures without altruism, voluntary participation, and punishment [33, 35]. Some similar conclusion has been found by Hanauske et al., Iqbal and Abbott, and Wu et al. ${ }^{[36-38]}$.

\section{B. Quantum dynamics and network models}

Busemeyer et al. set up a general quantum random walk model, and directly compare its assumptions with a general Markov random walk model. Their result indicate that the quantum model could help explain some paradoxical findings from the field of decision making such as the disjunction effect ${ }^{[39]}$. Iterated bipartite quantum games are implemented in terms of the discrete-time quantum walk on the line by Abal et al.. A quantum version of the well-known Prisoner's Dilemma bipartite game is presented as a specific example ${ }^{[40]}$. Romanell and Hernandez investigate the global chirality distribution of the quantum walk on the line when decoherence is introduced either through simultaneous measurements of the chirality and particle position, or as a result of broken links. The first mechanism drives the system towards a classical diffusive behavior; the second mechanism involves two different possibilities [12]. Chandrashekar and Banerjee present a new form of a Parrondo game using discrete-time quantum walk on a line. They present a quantum strategy for the players to cooperate by using their quantum coin operations alternatively and emerge as joint winner for situations where it is conditioned that the winner is decided only after even number of steps of walk evolution ${ }^{[29]}$.

\section{Quantum game applications}

Some papers study how to apply quantum game theory into society research. Based on the assumption that the main goal of scientists is the maximization of their reputation, Hanauske has performed modeling work for different possible game settings, namely a zero sum game, the prisoners' dilemma case and a version of the stag hunt game, which demonstrates the dilemma of scientists belonging to "non-open access communities". By extending the model using the quantum game theory approach, it can be seen that if the power of entanglement exceeds a certain value, the scientists will overcome the dilemma and terminate to publish only traditionally in all three settings ${ }^{[41]}$.Through the extension of the well-known hawk-dove game by a quantum approach, Hanauske et al. show that, depending on entanglement, evolutionary stable strategies also can emerge, which are not predicted by the classical evolutionary game theory and when the total economic population uses a non-aggressive quantum strategy [36] .
After proposing a quantum-like description of markets and economics, Piotrowski further presents a simple but nontrivial class of the quantum strategies in buying-selling games ${ }^{[42,43]}$. The Giffen paradoxes in quantum game model formalism is discussed by Sladkowski ${ }^{[44]}$.

Badredine explores the effects of non-independence on strategic interaction, where two types of non-independence effects are considered. The first one is the subjective nonindependence at the level of the individual actor by looking at how choice ambivalence shapes the decision-making process. The other is how inter-subjective non-independence across actors engenders collective strategies among two or more interacting actors ${ }^{[45]}$.

Based on the fact that service quality is the bottleneck for Chinese service industry, with the goal of consumer's perceived utility maximization, a classic game model and a quantum game model are proposed and established by Zhang and Xing. Through the research, their results show that the quantum game expands the strategy space of the firms, and can help them to obtain a superior equilibrium solution or better decision-making value than the classic game ${ }^{[46]}$.

\section{CONCLUSION AND FUTURE DEVELOPMENT}

\section{A. Basic elements of classical game and quantum game}

Quantum game theory is an exciting new topic that combines the physical behavior of information in quantum mechanical systems with game theory. The basic differences between the classical game and the quantum game are as follows $[11,14,23,27,47]$.

In classical game theory, game is defined as a set of players. A set of rules specify the possible actions of the players, and a set of payoff functions gives the rewards to the players for the various game outcomes. That is, a triple $\{N, \Omega, \Gamma\}$ where $N$ is the number of players; $\Omega=\left\{S_{1}, S_{2}, \ldots, S_{N}\right\}$ with $S_{j}$ being the set of strategies available to the $\boldsymbol{j t h}$ player; and $\Gamma=\left\{P_{1}, P_{2}, \ldots, P_{N}\right\}$ with $\boldsymbol{P}_{N}$ being the payoff function for the $\boldsymbol{j t h}$ player, $j=1, \ldots, N$.

Quantum games clearly describe and depict the processes of information exchanges among players and payoff realization, which can be formulated as $\boldsymbol{G}\{\boldsymbol{n}, \Theta(\mathrm{H}), \boldsymbol{\rho}, \boldsymbol{S}, \boldsymbol{u}\}$ where $\boldsymbol{n}$ is the number of players; $\mathrm{H}$ is the two dimensional Hilbert space; $\Theta(\mathrm{H})$ is the game's state space; $\rho \in \Theta(\mathrm{H}) \quad$ is the starting state; $S=S_{1} \times S_{2} \times \ldots \times S_{n}$ is the strategy space; and $u=u_{1} \times u_{2} \times \ldots \times u_{n}$ is the utility function with $\boldsymbol{u}_{\boldsymbol{i}}: \Theta(\mathrm{H}) \rightarrow \square$ for player $\boldsymbol{i}$. In quantum games, the state of the game can be represented by a qubit or tensor products of multiple qubits. 
Huang, Dingxuan \& Li, Shuliang. A Survey of the Current Status of Research on Quantum Games. In: Proceedings of the 2018 4th International Conference on Information Management (ICIM2018), 25th to 27th May 2018, Oxford, United Kingdom. IEEE. pp.46-52. ISBN: 978-1-5386-6146-8/18.

Put it simple, the classical games do its calculations in probability space, while quantum games operate in Hilbert space.

\section{B. Future development}

Although great effort has been made in the field of quantum game research, much still remains to be explored. None of the themes discussed above is mature, which leaves plenty of space for future work. This also opens avenue of quantum economics applications in various economic areas, such as public goods, correlated-value auctions, coordination games and digital rights management ${ }^{[48]}$.

Some papers in quantum games involve quantization of evolutionary games $[6,7,36]$. However, there is a noticeable limitation and constraint on the total number of players and participants within dynamic games. Numerous categories of dynamic games still remain unexplored, especially for those dynamic games with varied population size might benefit from such research. For instance, predator-prey model. Our modern society is a dynamic and open system. Therefore, combining quantum game theory with dynamic open system, can not only help us learn how the dynamic system changes in quantum situations, but also help us understand how the quantum entanglement and the payoff matrix influence the decision-making behavior of the decisionmaking agents.

\section{ACKNOWLEDGMENT}

This study is supported by the National Natural Science Foundation of China under grant No. 71662008, Sichuan 100 Talent Scheme grant (CHUAN ZU TONG [2012] No.142) (China), the National Natural Science Foundation of China under grant No. 71472158, the Key Research Institute of Philosophies and Social Sciences in Guangxi Universities (China) (15YB001, 16YB005), the Open fund from Guangxi Key Laboratory of New Energy and Building Saving (China) (Gui Ke Neng 16-J-21-8), the University of Westminster staff research allowances, UK.

\section{REFERENCES}

[1]Y. Li, G. Qin, X. Y. Zhou, and J. F. Du, "The application of asymmetric entangled states in quantum games," PHYSICS LETTERS A, vol. 355, p. 447-451, 2006.

[2]J. Eisert, M. Wilkens, and M. Lewenstein, "Quantum games and quantum strategies," PHYSICAL REVIEW LETTERS, vol. 83, p. 3077-3080, 1999.

[3]D. A. Meyer, "Quantum strategies," Physical Review Letters, vol. 82, p. 1052-1055, 1999.

[4]A. M. Kowalski, "Betting on dynamics," Physica A, vol. 458, p. 106-114, 2016.

[5]L. Pawela, "Quantum games on evolving random networks," PHYSICA A-STATISTICAL MECHANICS AND ITS APPLICATIONS, vol. 458, p. 179-188, 2016.
[6]L. Pawela and J. Sladkowski, "Cooperative quantum Parrondo's games," PHYSICA D-NONLINEAR PHENOMENA, vol. 256, p. 51-57, 2013.

[7]L. Pawela and J. Sladkowski, "Quantum Prisoner's Dilemma game on hypergraph networks," PHYSICA A-STATISTICAL MECHANICS AND ITS APPLICATIONS, vol. 392, p. 910917, 2013.

[8] A. Iqbal, J. M. Chappell, and D. Abbott, "Social optimality in quantum Bayesian games," PHYSICA A-STATISTICAL MECHANICS AND ITS APPLICATIONS, vol. 436, p. 798805, 2015.

[9]J. Dajka, D. Kloda, M. Lobejko, and J. Sladkowski, "Quantum Two Player Game in Thermal Environment," PLOS ONE, vol. $10,2015$.

[10]C. Hung, "Social networks, technology ties, and gatekeeper functionality: Implications for the performance management of R\&D projects," Research Policy, vol. 46, p. 305-315, 2017.

[11]H. Guo, J. H. Zhang, and G. J. Koehler, "A survey of quantum games," DECISION SUPPORT SYSTEMS, vol. 46, p. 318332, 2008.

[12]A. Romanelli and G. Hernandez, "Quantum walks: Decoherence and coin-flipping games," PHYSICA ASTATISTICAL MECHANICS AND ITS APPLICATIONS, vol. 390, p. 1209-1220, 2011.

[13]A. Iqbal and D. Abbott, "Constructing quantum games from a system of Bell's inequalities," PHYSICS LETTERS A, vol. 374, p. 3155-3163, 2010.

[14]A. P. Flitney and L. Hollenberg, "Nash equilibria in quantum games with generalized two-parameter strategies," PHYSICS LETTERS A, vol. 363, p. 381-388, 2007.

[15]A. Iqbal and A. H. Toor, "Quantum repeated games," PHYSICS LETTERS A, vol. 300, p. 541-546, 2002.

[16]A. Iqbal, T. Cheon, and D. Abbott, "Probabilistic analysis of three-player symmetric quantum games played using the Einstein-Podolsky-Rosen-Bohm setting," PHYSICS LETTERS A, vol. 372, p. 6564-6577, 2008.

[17]T. Ichikawa and I. Tsutsui, "Duality, phase structures, and dilemmas in symmetric quantum games," ANNALS OF PHYSICS, vol. 322, p. 531-551, 2007.

[18]L. Marinatto and T. Weber, "A quantum approach to static games of complete information," Physics Letters A, vol. 272, p. 291-303, 2000.

[19]J. Zhou, L. Ma, and Y. Li, "Multiplayer quantum games with continuous-variable strategies," PHYSICS LETTERS A, vol. 339, p. 10-17, 2005.

[20]H. Li, J. F. Du, and S. Massar, "Continuous-variable quantum games," PHYSICS LETTERS A, vol. 306, p. 73-78, 2002.

[21]Q. Chen, W. Yi, J. T. Liu, and K. L. Wang, "N-player quantum minority game," PHYSICS LETTERS A, vol. 327, p. 98-102, 2004.

[22]A. P. Flitney and A. D. Greentree, "Coalitions in the quantum minority game: Classical cheats and quantum bullies," PHYSICS LETTERS A, vol. 362, p. 132-137, 2007.

[23]A. P. Flitney, M. Schlosshauer, C. Schmid, W. Laskowski, and L. Hollenberg, "Equivalence between Bell inequalities and quantum minority games," PHYSICS LETTERS A, vol. 373, p. 521-524, 2009.

[24]C. D. Hill, A. P. Flitney, and N. C. Menicucci, "A competitive game whose maximal Nash-equilibrium payoff requires quantum resources for its achievement," PHYSICS LETTERS A, vol. 374, p. 3619-3624, 2010. 
Huang, Dingxuan \& Li, Shuliang. A Survey of the Current Status of Research on Quantum Games. In: Proceedings of the 2018 4th International Conference on Information Management (ICIM2018), 25th to 27th May 2018, Oxford, United Kingdom. IEEE. pp.46-52. ISBN: 978-1-5386-6146-8/18.

[25]O. G. Zabaleta, J. P. Barrangu, and C. M. Arizmendi, "Quantum game application to spectrum scarcity problems," PHYSICA A-STATISTICAL MECHANICS AND ITS APPLICATIONS, vol. 466, p. 455-461, 2017.

[26]A. P. Flitney and D. Abbott, "Quantum models of Parrondo's games," PHYSICA A-STATISTICAL MECHANICS AND ITS APPLICATIONS, vol. 324, p. 152-156, 2003.

[27]A. P. Flitney, J. Ng, and D. Abbott, "Quantum Parrondo's games," PHYSICA A-STATISTICAL MECHANICS AND ITS APPLICATIONS, vol. 314, p. 35-42, 2002.

[28]L. Chen, C. F. Li, M. Gong, and G. C. Guo, "Quantum Parrondo game based on a quantum ratchet effect," PHYSICA A-STATISTICAL MECHANICS AND ITS APPLICATIONS, vol. 389, p. 4071-4074, 2010.

[29]C. M. Chandrashekar and S. Banerjee, "Parrondo's game using a discrete-time quantum walk," PHYSICS LETTERS A, vol. 375, p. 1553-1558, 2011

[30]Y. F. Zhu, N. G. Xie, Y. Ye, and F. R. Peng, "Quantum game interpretation for a special case of Parrondo's paradox," PHYSICA A-STATISTICAL MECHANICS AND ITS APPLICATIONS, vol. 390, p. 579-586, 2011.

[31]L. Wang, N. G. Xie, Y. F. Zhu, Y. Ye, and R. Meng, "Parity effect of the initial capital based on Parrondo's games and the quantum interpretation," PHYSICA A-STATISTICAL MECHANICS AND ITS APPLICATIONS, vol. 390, p. 45354542, 2011.

[32]R. Li, Y. F. Zhu, J. Y. Guo, L. Wang, and N. G. Xie, "The quantum game interpretation for a special phenomenon of Parrondo's paradox,". vol. 15 AMSTERDAM: ELSEVIER SCIENCE BV, 2011.

[33]A. Iqbal and A. H. Toor, "Evolutionarily stable strategies in quantum games," PHYSICS LETTERS A, vol. 280, p. 249256, 2001.

[34]R. Kay, N. F. Johnson, and S. C. Benjamin, "Evolutionary quantum game," JOURNAL OF PHYSICS AMATHEMATICAL AND GENERAL, vol. 34, p. L547-L552, 2001.

[35]Z. Y. Liao, G. Qin, L. Z. Hu, S. J. Li, N. Y. Xu, and J. F. Du, "Static and evolutionary quantum public goods games," PHYSICS LETTERS A, vol. 372, p. 3586-3590, 2008.

[36]M. Hanauske, J. Kunz, S. Bernius, and W. Konig, "Doves and hawks in economics revisited: An evolutionary quantum game theory based analysis of financial crises," PHYSICA ASTATISTICAL MECHANICS AND ITS APPLICATIONS, vol. 389, p. 5084-5102, 2010.
[37]A. Iqbal and D. Abbott, "Non-factorizable joint probabilities and evolutionarily stable strategies in the quantum prisoner's dilemma game," Physics Letters A, vol. 373, p. 2537-2541, 2009.

[38]Q. Y. Wu, L. C. Jiao, Y. Y. Li, and X. Z. Deng, "A novel quantum-inspired immune clonal algorithm with the evolutionary game approach," PROGRESS IN NATURAL SCIENCE-MATERIALS INTERNATIONAL, vol. 19, p. 1341-1347, 2009.

[39]J. R. Busemeyer, Z. Wang, and J. T. Townsend, "Quantum dynamics of human decision-making," Journal of Mathematical Psychology, vol. 50, p. 220-241, 2006.

[40]G. Abal, R. Donangelo, and H. Fort, "Conditional strategies in iterated quantum games," PHYSICA A-STATISTICAL MECHANICS AND ITS APPLICATIONS, vol. 387, p. 53265332, 2008.

[41]M. Hanauske, S. Bernius, and B. Dugall, "Quantum game theory and open access publishing," PHYSICA ASTATISTICAL MECHANICS AND ITS APPLICATIONS, vol. 382, p. 650-664, 2007.

[42]E. W. Piotrowski and J. Sladkowski, "Quantum market games," PHYSICA A-STATISTICAL MECHANICS AND ITS APPLICATIONS, vol. 312, p. 208-216, 2002.

[43]E. W. Piotrowski, "Fixed point theorem for simple quantum strategies in quantum market games," PHYSICA ASTATISTICAL MECHANICS AND ITS APPLICATIONS, vol. 324, p. 196-200, 2003.

[44]J. Sladkowski, "Giffen paradoxes in quantum market games," PHYSICA A-STATISTICAL MECHANICS AND ITS APPLICATIONS, vol. 324, p. 234-240, 2003.

[45]B. Arfi, "Quantum social game theory," PHYSICA ASTATISTICAL MECHANICS AND ITS APPLICATIONS, vol. 374, p. 794-820, 2007.

[46]C. H. Zhang and P. Xing, "A research on service quality decision-making of Chinese communications industry based on quantum game," PHYSICA A-STATISTICAL MECHANICS AND ITS APPLICATIONS, vol. 432, p. 9-15, 2015.

[47]A. P. Flitney and D. Abbott, "Advantage of a quantum player over a classical one in $2 \mathrm{X} 2$ quantum games," PROCEEDINGS OF THE ROYAL SOCIETY A-MATHEMATICAL PHYSICAL AND ENGINEERING SCIENCES, vol. 459, p. 2463-2474, 2003.

[48]K. Chen and T. Hogg, "How Well Do People Play a Quantum Prisoner's Dilemma," Quantum Information Processing, vol. 5, p. 43-67, 2007. 